\title{
On a class of exact solutions to the Fokker-Planck equations
}

\author{
L. Garrido and J. Masoliver \\ Departamento de Física Teórica, Universidad de Barcelona, Diagonal, 647, Barcelona-28, Spain
}

(Received 9 December 1981; accepted for publication 19 February 1982)

In this paper we study under which circumstances there exists a general change of gross

variables that transforms any Fokker-Planck equation into another of the Ornstein-Uhlenbeck

class that, therefore, has an exact solution. We find that any Fokker-Planck equation will be exactly solvable by means of a change of gross variables if and only if the curvature tensor and the torsion tensor associated with the diffusion is zero and the transformed drift is linear. We apply our criteria to the Kubo and Gompertz models.

PACS numbers: 05.30. - d

\section{INTRODUCTION}

The Fokker-Planck equation (FPE) describes a generalized diffusion process for the probability density $P(q, t)$ $=P\left(q^{1}, \ldots q^{n} ; t\right)$ in an $n$-dimensional physical space spanned by the $n$ gross variables $\left\{q^{\nu}, v=1, \ldots, n\right\}$. If $D^{\mu \nu}(q)$ and $f^{\mu}(q)$ are the diffusion matrix and the drift, the FPE reads

$$
\dot{P}(q, t)=-\partial_{\mu}\left[f^{\mu}(q)-\frac{1}{2} \partial_{v} D^{\mu \nu}(q)\right] P(q, t)
$$

when $\partial_{\mu}$ means $\partial / \partial q^{\mu}$.

It has been possible to find an exact solution to the FPE when the diffusion is constant and when the drift is linear. ${ }^{1}$ There exists also a class of FPE's that have exact solution and whose model is an FPE with linear drift and diffusion $\delta^{\mu \nu}{ }^{2}$ Any FPE of this class can be reduced to its model by means of a change in the gross variables. We may ask ourselves immediately the following question: Under which conditions does there exist a change of gross variables that transforms any FPE into another FPE with linear drift and diffusion $\delta^{\mu \nu}$, i.e., that has an exact solution? This last question has a complete answer and constitutes the central result of this paper, in which we intend to give the necessary and sufficient conditions to determine if any FPE has an exact solution related with the exact solution of the Ornstein-Uhlenbeck process by means of a general change of gross variables.

In Sec. II we show how any FPE can be transformed into another one with exact solution. We use here the covariant formulation of $\mathrm{FPE}^{3,4}$ by means of which we obtain a clear and rigorous method for such a transformation. In Sec. III we give the necessary and sufficient conditions to be satisfied by our original FPE so that such a transformation of gross variables exists: the curvature and torsion tensors have to be zero. These criteria characterized with precision a class of FPE's that have exact solutions. ${ }^{2}$ In Sec. IV we study some subclasses of FPE that have physical importance, among which we find the Kubo ${ }^{5}$ and Gompertz ${ }^{6}$ models.

In this paper we suppose the sum over repeated indices except those that are within a parenthesis.

\section{TRANSFORMATION OF AN FPE INTO ANOTHER ONE WHOSE DIFFUSION IS $\delta^{\mu \nu}$}

Let $M$ be the manifold formed by the physical states of the system. ${ }^{7}$ This manifold is characterized by two sets of gross variables, $\left\{q^{\mu}\right\}$ and $\left\{q^{\mu}\right\}$, related among themselves by means of continuous and differentiable functions and which conserve the number of gross variables. The diffusion tensor $D^{\mu \nu}(q)$ is the metric tensor of the manifold.

In this section we are going to transform a general diffusion process (1.1) into an FPE whose diffusion matrix is $\delta^{\mu \nu}$. This last equation has exact solution if the transformed drift is linear (Ornstein-Uhlenbeck process). We write Eq. (1.1) covariantly by ${ }^{4}$

$$
\begin{aligned}
\dot{S}(q, t)= & -\nabla_{\mu}\left(h^{\mu} S-\frac{1}{2} D^{\mu \nu} \nabla_{\mu} S\right) \\
& -\frac{1}{2} D^{\sigma \tau} \nabla_{\nu} D_{\tau \sigma}\left(h^{\nu} S-\frac{1}{2} D^{\mu \nu} \nabla_{\mu} S\right),
\end{aligned}
$$

where $\nabla_{\mu}$ is the covariant derivative of the Riemann connection of $D^{\mu v 8}$

$$
\nabla_{\mu} h^{v}=\partial_{\mu} h^{v}+\Gamma_{\rho \mu}^{v} h^{\rho}
$$

where $\Gamma_{\rho \mu}^{v}$ are the Christoffel symbols

$$
\Gamma_{\rho \mu}^{v}=\frac{1}{2} D^{v \sigma}\left(\partial_{\mu} D_{\sigma \rho}+\partial_{\rho} D_{\sigma \mu}-\partial_{\sigma} D_{\rho \mu}\right) \text {. }
$$

The function $S(q, t)$ is the scalar probability density

$$
S(q, t)=P(q, t) D^{1 / 2} \quad\left(D=\operatorname{det} D^{\mu v}\right)
$$

the vector $h(q)$ is the covariant drift ${ }^{4}$

$$
\begin{aligned}
& h^{v}(q)=f^{\nu}(q)-\frac{1}{2} D^{1 / 2} \partial_{\mu}\left(D^{\mu \nu} / D^{1 / 2}\right), \\
& D_{\mu \nu}=\left(D^{-1}\right)^{\mu \nu} .
\end{aligned}
$$

Let $\left\{q^{\prime v}\right\}$ be the new "gross variables" related to the old ones by means of

$$
J_{\rho}^{\sigma}=\frac{\partial q^{\prime \sigma}}{\partial q^{\rho}}, \quad J_{\sigma}^{-1 \rho}=\frac{\partial q^{\rho}}{\partial q^{\prime \sigma}},
$$

which obviously satisfies

$$
\partial_{\sigma} J_{\mu}^{v}=\partial_{\mu} J_{\sigma}^{v} \text {. }
$$

Let us suppose that the new variables $\left\{q^{v}\right\}$ are such that $D^{\mu v}=\delta^{\mu v}$.

The corresponding FPE is

$$
\dot{P}^{\prime}\left(q^{\prime}, t\right)=-\partial_{\mu}^{\prime}\left[\left(f^{\prime \mu}\left(q^{\prime}\right)-\frac{1}{2} \partial_{\mu}^{\prime}\right] P^{\prime}\left(q^{\prime}, t\right)\right.
$$

since for the Euclidean metric $\delta^{\mu v}$ the Christoffel symbols are zero and besides

$$
\begin{aligned}
& S^{\prime}\left(q^{\prime}, t\right)=P^{\prime}\left(q^{\prime}, t\right) \equiv P\left(q\left(q^{\prime}\right), t\right), \\
& h^{\prime \mu}\left(q^{\prime}\right)=f^{\prime \mu}\left(q^{\prime}\right) \equiv f^{\mu}\left(q\left(q^{\prime}\right)\right) .
\end{aligned}
$$

Remembering that $f^{\prime \mu}=h^{\prime \mu}=J_{v}{ }^{\mu} h^{\nu}$, the transformed drift 
is

$$
f^{\prime \mu}=J_{v}{ }^{\mu}\left(f^{\nu}+\frac{1}{2} D^{\alpha \beta} \Gamma_{\alpha \beta}^{\nu}\right) .
$$

The transformed FPE (2.10) is exactly solvable if it represents an Ornstein-Uhlenbeck process. The solution of $(2.10)$ with the initial condition

$$
P^{\prime}\left(q^{\prime}, 0\right)=\delta^{n}\left(q^{\prime}-q_{0}\right)
$$

is $^{1}$

$$
\begin{aligned}
P^{\prime}\left(q^{\prime}, t\right)= & {\left[\Pi^{n} \operatorname{det} \sigma_{\mu \nu}(t)\right]^{-1 / 2} } \\
& \times \exp \left(-\sigma_{\mu \nu}^{-1}(t)\left[q^{\prime \mu}-q^{\prime \mu}(t)\right]\left[q^{\prime \nu}-q^{\prime \nu}(t)\right]\right\}
\end{aligned}
$$

if from the transformed linear drift

$$
f^{\prime \mu}\left(q^{\prime}\right)=A^{\mu}{ }_{\nu} q^{\prime \nu}+B^{\mu}
$$

we define

$$
q^{\prime \mu}(t) \equiv \gamma_{\rho}^{\mu}(t)\left[q_{0}^{\rho}+A_{z}^{-1 \rho} B^{z}\right]-A_{\rho}^{-1 \mu} B^{\rho},
$$

where $\gamma_{\rho}^{\mu}(t)$ is defined by

$$
\begin{aligned}
& \dot{\gamma}_{\rho}^{\mu}(t)=A_{\alpha}^{\mu} \gamma_{\rho}^{\alpha}(t), \\
& \gamma_{\rho}^{\mu}(0)=\delta_{\rho}^{\mu},
\end{aligned}
$$

and

$$
\begin{aligned}
& \sigma_{\mu \nu}(t)=\left\{\gamma_{\mu}^{\rho}(0) \gamma_{\nu}^{\tau}(0)-\gamma_{\mu}^{\rho}(t) \gamma_{\nu}^{\tau}(t)\right\} \Omega_{\rho \tau} \\
& A_{\mu}^{\alpha} \Omega^{\nu}{ }_{\alpha}+\Omega^{\alpha}{ }_{\mu} A_{\alpha}^{\nu}=-2 \delta^{\nu}{ }_{\mu}
\end{aligned}
$$

Therefore, if there exists a system of "gross variables" $\left\{q^{\prime v}\right\}$ that satisfies Eqs. (2.9) and (2.15), the solution of the original FPE is (2.14) when we substitute, for $q^{\prime}, q^{\prime}(q)$ and, for $P\left(q^{\prime}(q), t\right), P(q, t) D^{1 / 2}$.In thiscase the integrations of the FPE is simply reduced to the integration of the change of variables (2.7).

\section{NECESSARY AND SUFFICIENT CONDITIONS TO SOLVE EXACTLY AN FPE BY MEANS OF A CHANGE OF VARIABLES}

There will not always exist a change of variables $q=q\left(q^{\prime}\right)$ such that $D^{\prime \mu \nu}=\delta^{\mu \nu}$. In the Appendix we show that the necessary and sufficient conditions, in order that $D^{\mu \nu}=\delta^{\mu \nu}$, are that the curvature tensor $R_{\mu \nu \alpha \beta}$ and torsion tensor $T_{v \alpha}^{\mu}{ }^{8}$ associated with the diffusion matrix be zero.

Therefore the conditions

$$
\begin{aligned}
R_{\mu v \alpha \beta}= & \frac{1}{2}\left(\partial_{\nu \alpha}^{2} D_{\mu \beta}+\partial_{\mu \beta}^{2} D_{v \alpha}-\partial_{\nu \beta}^{2} D_{\mu \alpha}-\partial_{\mu \alpha}^{2} D_{v \beta}\right) \\
& \quad+D_{\sigma \rho}\left(\Gamma_{v \alpha}^{\sigma} \Gamma_{\mu \beta}^{\rho}-\Gamma_{\nu \beta}^{\sigma} \Gamma_{\mu \alpha}^{\rho}\right)=0 \\
T_{v \sigma}{ }^{\mu}= & \Gamma_{\nu \alpha}^{\mu}-\Gamma_{\alpha v}^{\mu}=0
\end{aligned}
$$

guarantee the existence of a change of variables determined by the matrix $J_{\lambda}{ }^{\mu}$ such that in the new variables the diffusion matrix $D^{\prime \mu \nu}$ becomes $\delta^{\mu \nu}$. If we work with Riemann's connection $D^{\mu \nu}$, the Christoffel symbols are given by Eq. (2.3), and condition (3.2) is satisfied identically.

In the same appendix [Eq. (A3)] we show that the matrix $J_{\lambda}{ }^{\mu}$ for the variables transformations must satisfy the relation

$$
\partial_{\mu} J_{v}^{\lambda}=\Gamma_{\mu \nu}^{\alpha} J_{\alpha}^{\lambda} .
$$

For this equation the formal solution is

$$
J_{v}{ }^{\lambda}(q)=S^{\alpha}{ }_{v}\left(q, q_{0}\right)_{\alpha}{ }^{\lambda}\left(q_{0}\right)
$$

where

$$
S^{\alpha}{ }_{\nu}\left(q, q_{0}\right) \equiv \exp \left[\int_{q_{0}}^{q} \Gamma_{\mu \nu}^{\alpha}\left(q^{\prime \prime}\right) d q^{\mu \mu}\right] .
$$

On this formal solution we should impose "initial conditions" that are not given here.

The diffusion matrix in the variables $\left\{q^{\prime \nu}\right\}$ becomes a constant matrix; therefore, the general solution of (3.4) should satisfy:

$$
J_{\mu}^{-1 \sigma} J_{v}^{-1 \rho} D^{\mu \nu}(q)=A^{\sigma \rho}
$$

or, equivalently,

$$
J_{\mu}^{\sigma} J_{\nu}^{\rho} A_{\sigma \rho}=D_{\mu \nu}(q),
$$

where $A_{\sigma \rho}$ is a constant matrix. Concretely, we could take $A_{\sigma \rho}=\delta_{\sigma \rho}$; in this case the transformation matrix satisfies

$$
D_{\mu \nu}(q)=J_{\mu}^{\sigma} J_{\nu}^{\rho} \delta_{\sigma \rho}
$$

Relation (3.8) is used in Ref. 2 to characterize a whole class of FPE that have exact solutions.

In order that the transformed FPE represent an Ornstein-Uhlenbeck process, which has an exact solution, it is also necessary that the transformed $\operatorname{drift} f^{\prime \mu}\left(q^{\prime}\right)$ be linear, i.e.,

$$
\partial_{\sigma \lambda}^{2} f^{\prime \mu}\left(q^{\prime}\right)=0 \text {. }
$$

In the coordinates $q^{\prime}$ the covariant derivation $\nabla^{\prime}$ coincides with the ordinary derivatives and besides the $\operatorname{drift} f^{\prime \mu}$ is the covariant drift $h^{\prime \mu}$. Therefore, the covariant expression for (3.9) is

$$
\nabla_{\gamma}^{\prime} \nabla_{\gamma}^{\prime} h^{\prime \mu}=0
$$

which if written in the original variables becomes

$$
\nabla_{\gamma} \nabla_{\lambda} h^{\mu}=0 \text {. }
$$

Let us observe that the condition (3.11) does not require a knowledge of the change of gross variables: it is sufficient to know the Christoffel symbols $\Gamma_{v \alpha}^{\mu}$ that can be evaluated differentiating the diffusion $D^{\mu \nu}$.

From what has been said above, we can check whether or not any FPE (1.1) represents, in a certain set of gross variables, an Ornstein-Uhlenbeck process that has an exact solution. We could test if the diffusion matrix $D^{\mu v}$ satisfies the condition (3.1) and if the drift satisfies the condition (3.11). If the answer is affirmative, we can integrate the change of variables by means of a matrix $J_{\mu}^{\sigma}$ of the type (3.7). The solution of the FPE is the one transformed from Eq. (2.14).

\section{APPLICATIONS}

An important case are those processes whose diffusion is diagonal:

$$
D^{\mu v}(q)=D_{(\mu)}(q) \delta^{\mu v} .
$$

The only elements of curvature tensor that are not zero are $\boldsymbol{R}_{\mu \nu \mu \rho}$ and $\boldsymbol{R}_{\mu \nu \mu \nu}$. As it is easy to check a diffusion matrix of the form

$$
D^{\mu \nu}(q)=\varphi_{(\mu)}\left(q_{\mu}\right) \delta^{\mu \nu}
$$

with $\varphi_{(\mu)}\left(q_{\mu}\right)>0$ satisfies simultaneously the conditions

$$
R_{\mu v \mu \rho}=0 \text {, }
$$




$$
R_{\mu \nu \mu \nu}=0 .
$$

The corresponding matrix of the change of gross variables is any solution of the equations

$$
\begin{aligned}
& \partial_{\mu} J_{\nu}^{\rho}=0 \quad(\mu \neq v), \\
& \partial_{v} J_{v}^{\rho}=\frac{1}{2} \partial_{v} \ln \varphi_{(v)} J_{v}^{\rho} .
\end{aligned}
$$

The general solution of $(4.5)$ and (4.6) is

$$
J_{v}^{\lambda}=M_{\beta}^{\lambda}\left(\varphi_{(\beta)}\left(q_{\beta}\right)\right)^{1 / 2} \delta^{\beta}{ }_{v},
$$

where $M_{B}^{\lambda}$ is a nonsingular constant matrix.

With the metric (4.2) the covariant drift in its original variables is

$$
h^{\mu}=f^{\mu}+\frac{1}{4} \varphi_{(\mu)}^{-1} \partial_{\mu} \varphi_{(\mu)},
$$

and the condition that the original drift $f^{\mu}(q)$ should satisfy in order that the transformed $\operatorname{drift} f^{\prime \nu}\left(q^{\prime}\right)$ be linear is

$$
\nabla_{\lambda} \nabla_{\gamma}\left(f^{\mu}+\frac{1}{4} \varphi_{(\mu)}^{-2} \partial_{\mu} \varphi_{(\mu)}\right)=0 .
$$

To the subclass of models represented by Eq. (4.2) belong the Kubo and Gompertz models. Let the function $\varphi_{(\mu)}\left(q_{\mu}\right)$ be of the form

$$
\varphi_{(\mu)}\left(q_{\mu}\right)=a_{(\mu)}^{2} q_{(\mu)}^{2} ;
$$

if the transformed drift is constant, we have $n$ monodimensional Kubo models.

Similarly, if we have

$$
\varphi_{(\mu)}\left(q_{\mu}\right)=a_{(\mu)}^{2} q_{(\mu)}^{2}\left|\log \left(b_{(\mu)}\right) q_{(\mu)}\right|^{2}
$$

when the transform drift is constant, we obtain $n$ monodimensional Gompertz models. ${ }^{2,6}$

Another example is the case when the diffusion matrix is conformally flat:

$$
D^{\mu \nu}(q)=D(q) \delta^{\mu \nu}
$$

with $D(q)>0$. In such a case there will exist a change of variables such that $D^{\prime \mu \nu}=\delta^{\mu v}$ if the function $D(q)$ satisfies the equations

$$
\begin{array}{r}
3 D^{-1} \partial_{\nu} D \partial_{\rho} D-2 \partial_{\rho v}^{2} D=0 \quad(\rho \neq v), \\
D^{-1}\left[\left(\partial_{(\mu)} D\right)^{2}+\left(\partial_{(v)} D\right)^{2}-\frac{1}{2} \sum_{\sigma \neq \mu, \nu}\left(\partial_{\sigma} D\right)^{2}\right] \\
-\left(\partial_{(\mu)(\mu)}^{2} D+\partial_{(\nu)(v)} D\right)=0 \quad(\mu \neq v) .
\end{array}
$$

A solution of these equations is

$$
D(q)=A \exp \left[B\left(q_{\mu}+q_{\nu}\right)+c\right]
$$

with an associated transformation matrix

$$
J_{\sigma}^{\rho}=A^{1 / 2} \exp \left\{\frac{1}{2} B\left(q_{\mu}+q_{v}\right)+c\right\} M_{\sigma}^{\rho} .
$$

\section{CONCLUSIONS}

Using the powerful methods of the differential geometry we have found a class of FPE that are exactly solvable by means of change of variables.

Choosing as the metric tensor of the physical space the diffusion matrix, the corresponding FPE will be exactly solvable by means of a change of gross variables if and only if the curvature tensor and the torsion tensor associated with the diffusion is zero, and the transformed drift is linear.

To write the solution of the FPE in the original variables, we have to know the functions $q^{\prime}=q^{\prime}(q)$ of the change of variables and substitute them in the solution (2.14). Therefore, with the method presented in this paper the solution of an FPE is reduced to integrating a change of gross variables. Anyway, with the method presented above we can test any FPE to see whether or not it has an exact solution by means of a change of coordinates: The diffusion should satisfy Eqs. (3.1) and (3.2) and the drift equation (3.11).

And so to write the solution of the FPE in the original variables, we should know the functions of such a change of variables $q^{\prime}=q^{\prime}(q)$ and enter into the solution (2.14). Therefore, the integration of an FPE equation is reduced with the method presented here to the integration of a change of variables.

\section{ACKNOWLEDGMENTS}

One of us (J. M.) would like to thank Dr. Llosa for his help and his useful suggestions.

\section{APPENDIX}

We are going to show that the necessary and sufficient conditions which makes that the metric $D^{\mu \nu}(q)$ (diffusion matrix) become Euclidean in some coordinate system are that the associated curvature tensors and the torsion be zero.

Indeed, let us suppose that there exists a coordinate system $q^{\prime \alpha}=q^{\prime \alpha}(q)$ such that $D^{\prime \mu \nu}\left(q^{\prime}\right)=\delta^{\mu \nu}$; in this case the Christoffel symbols of the metric $D^{\prime}$ are zero:

$$
\Gamma_{\mu \nu}^{\alpha}\left(q^{\prime}\right)=0 \text {. }
$$

Since the relation beween the Christoffel symbol for different coordinates system is given by ${ }^{8}$

$$
\begin{aligned}
\Gamma_{\mu \nu}^{\alpha}(q)= & \Gamma_{\rho \sigma}^{\prime \lambda}\left(q^{\prime}\right) \frac{\partial q^{\alpha}}{\partial q^{\prime \lambda}} \frac{\partial q^{\prime \rho}}{\partial q^{\mu}} \frac{\partial q^{\prime \sigma}}{\partial q^{\nu}} \\
& +\frac{\partial^{2} q^{\prime \lambda}}{\partial q^{\mu} \partial q^{\nu}} \frac{\partial q^{\alpha}}{\partial q^{\prime \lambda}}
\end{aligned}
$$

we will have

$$
\frac{\partial q^{\prime \lambda}}{\partial q^{\alpha}} \Gamma_{\mu \nu}^{\alpha}(q)=\frac{\partial^{2} q^{\prime \lambda}}{\partial q^{\mu} \partial q^{\nu}} .
$$

By means of Eq. (2.7), the relation (A2) may be written as

$$
J_{\alpha}^{\lambda} \Gamma_{\mu \nu}^{\alpha}(q)=\partial_{\mu} J_{\nu}^{\lambda} .
$$

If there exists a system of coordinates $\left\{q^{\prime \nu}\right\}$ such that $D^{\prime \mu \nu}\left(q^{\prime}\right)=\delta^{\mu \nu}$, or, equivalently, such that $\Gamma_{\mu \nu}^{\prime \alpha}\left(q^{\prime}\right)=0$, there will exist a transformation matrix solution of Eq. (A3). Therefore, (A3) should satisfy the integrability equations

$$
\partial_{\rho \mu}^{2} J_{\mu}^{\lambda}-\partial_{\mu \rho}^{2} J_{v}^{\lambda}=0
$$

Deriving (A3) and entering in (A4), we conclude that $J$ exists if

$$
\begin{aligned}
J_{\alpha}^{\lambda}\left[\partial_{\rho} \Gamma_{\mu \nu}^{\alpha}(q)-\partial_{\mu} \Gamma_{\rho \nu}^{\alpha}(q)\right] & \\
& +J_{\beta}^{\lambda}\left[\Gamma_{\rho \alpha}^{\beta}(q) \Gamma_{\mu \nu}^{\alpha}(q)-\Gamma_{\mu \nu}^{\beta}(q) \Gamma_{\rho \nu}^{\alpha}(q)\right]=0
\end{aligned}
$$

this condition is satisfied if

$$
R_{\mu \nu \rho}^{\beta} \equiv \partial_{\rho} \Gamma_{\mu \nu}^{\beta}-\partial_{\mu} \Gamma_{\rho \nu}^{\beta}+\Gamma_{\rho \alpha}^{\beta} \Gamma_{\mu \nu}^{\alpha}-\Gamma_{\mu \alpha}^{\beta} \Gamma_{\rho \nu}^{\alpha}=0,
$$

where $R_{\mu \nu \rho}^{\beta}$ is the curvature tensor of the manifold. ${ }^{8}$ And, 
since

$$
R_{\alpha \mu \nu \rho} \equiv D_{\alpha \beta} R_{\mu \nu \rho}^{\beta}=0,
$$

the matrix $J_{\alpha}^{\lambda}$ exists if all components of the curvature tensor are zero. But we could ask ourselves whether or not this matrix corresponds to a continuous and differentiable change of gross variables, i.e., if we can write $J_{\alpha}{ }^{\lambda}=\partial_{\alpha} q^{\lambda}$. The well-known condition

$$
\partial_{\mu} J_{\alpha}^{\lambda}=\partial_{\alpha} J_{\mu}^{\lambda}
$$

should then be satisfied, which is equivalent to

$$
J_{\beta}^{\lambda}\left(\Gamma_{\mu \alpha}^{\beta}-\Gamma_{\alpha \mu}^{\beta}\right)=0,
$$

as can be seen making use of (A3). This implies that the torsion tensor is zero

$$
T_{\mu \alpha}^{\beta} \equiv \Gamma_{\mu \alpha}^{\beta}-\Gamma_{\alpha \mu}^{\beta}=0 .
$$

'H. Haken, Rev. Mod. Phys. 47, 67 (1975).

${ }^{2}$ M. San Miguel, Z. Phys. B 33, 307 (1979)

${ }^{13}$ R. Graham, Z. Phys. B 26, 397 (1977).

${ }^{4}$ L. Garrido, Physica A 100, 140 (1980).

${ }^{5}$ W. Horsthemke and A. Bach, Z. Phys. B 22, 189 (1975).

${ }^{6}$ H. Haken, Z. Phys. B 24, 321 (1976).

${ }^{7} \mathrm{~L}$. Garrido and J. Llosa, "The intrinsic Fokker-Planck equation," in Systems Far from Equilibrium, edited by L. Garrido (Springer-Verlag, New York, 1980).

${ }^{8}$ L. D. Landau and E. M. Lifshitz, Teoría clasica de campos (Reverté, Barcelona, 1966); S. Weinberg, Gravitation and Cosmology (Wiley, New York, 1972); N. J. Hicks, Notes on Differential Geometry (Van Nostrand, London, 1974). 